\title{
Regular and Chaotic Regimes in Scalar Field Cosmology
}

\author{
Alexey V. TOPORENSKY \\ Sternberg Astronomical Institute, Moscow University, Moscow, 119899 Russia \\ E-mail: lesha@sai.msu.ru
}

Received October 25, 2005, in final form March 10, 2006; Published online March 21, 2006

Original article is available at http://www.emis.de/journals/SIGMA/2006/Paper037/

\begin{abstract}
A transient chaos in a closed FRW cosmological model with a scalar field is studied. We describe two different chaotic regimes and show that the type of chaos in this model depends on the scalar field potential. We have found also that for sufficiently steep potentials or for potentials with large cosmological constant the chaotic behavior disappears.
\end{abstract}

Key words: cosmology; scalar field; chaotic dynamics

2000 Mathematics Subject Classification: 37B25; 83C75

\section{Introduction}

The phenomenon of a transient chaos (when a dynamical system behaves chaotically during a transient period before reaching some other regime) becomes in last two decade a matter of intense investigations. More than 20 years ago it was remarked that a periodic attractor may follow a temporal chaotic behavior (see [1] and references therein). After, this kind of chaos has been found in a broader class of dynamical systems which have no attractors at all. In the paper of Gaspard and Rice [2] the dynamics generated by scattering of a small disk particle on three hard discs in a plane was described. For an arbitrary initial data apart from a zero measure set this particle leaves the system after some time interval (which can be arbitrarily large), during which the small disc has a chain of scattering on the three discs.

Several years earlier this kind of behavior was discovered in a cosmological model describing the evolution of a closed Friedman-Robertson-Walker (FRW) Universe filled with a massive scalar field [3]. The analog of scattering on a disc in this model is "bounce" - a transition from a cosmological collapse to a cosmological expansion of the Universe. The final regime of the dynamics for almost all initial condition is falling into a cosmological singularity. The description of the set of periodic trajectories in this model in the language of symbolic dynamics and calculation of topological entropy have been done in [4]. Later, such analysis was done for cosmological models with other types of a scalar field $[5,6,7]$. It appears that depending on the particular form of the scalar field potential, the dynamics may be either chaotic or regular. Several types of transition from chaos to a non-chaotic dynamics for particular one-parameter families of potential were described in [7]. In the present paper we summarize our knowledge on possible regimes in closed FRW cosmology with a scalar field.

\section{Equations of motion and basic properties}

We study the following ODE system (the derivation see in [7]):

$$
\frac{m_{P}^{2}}{16 \pi}\left(\ddot{a}+\frac{\dot{a}^{2}}{2 a}+\frac{1}{2 a}\right)+\frac{a \dot{\varphi}^{2}}{8}-\frac{a V(\varphi)}{4}=0,
$$




$$
\ddot{\varphi}+\frac{3 \dot{\varphi} \dot{a}}{a}+V^{\prime}(\varphi)=0 .
$$

with two variables - a scale factor $a$ and a scalar field $\varphi$. Here $m_{P}$ is a constant fixed parameter the Planck mass, the scalar field potential $V(\phi)$ is a smooth nonnegative function with $V(0)=0$. The ratio $H \equiv \dot{a} / a$ is called the Hubble parameter.

This system has one first integral of motion

$$
-\frac{3}{8 \pi} m_{P}^{2} a\left(\dot{a}^{2}+1\right)+\frac{a^{3}}{2}\left(\dot{\varphi}^{2}+2 V(\varphi)\right)=C .
$$

This integral play the role of energy and is equal to zero for cosmological solutions of the system (1), (2). This property arises from the known fact that in General Relativity the Hamiltonian vanishes identically. That is why in this section we restrict ourself by $C=0$ three-dimensional hypersurface in the whole 4-dimensional phase space of (1), (2) leaving the general situation to the Section 4.

The equation (2) for the scalar field looks like the equation for a harmonic oscillator with a time dependent "friction" $3 H$ which is positive in an expanding Universe. One of the most important in modern cosmology, the slow-roll regime can occurs when this "friction" is much larger than the frequency of the oscillator. More precisely, slow-roll approximation is characterized by the system

$$
H=\sqrt{\frac{8 \pi}{3 m_{P}^{2}} V(\varphi)}, \quad \dot{\varphi}=\frac{V^{\prime}(\varphi)}{3 H},
$$

resulting by neglecting second derivative terms, kinetic energy of the scalar field and spatial curvature. This regime in rather natural for physically admissible initial conditions $[9,10,11]$ and leads to fast growth of the scale factor $a$ while the scalar field $\varphi$ slow rolls toward zero. When the scalar field $\varphi$ falls below some value $\varphi \sim m_{P}$ this regime disappears [8].

In the opposite case, when the "friction" is small, the dynamics of $\varphi$ is damping oscillations [12]. This regime is typical for late time evolution of the Universe. However, in contrast to zero- or negative spatial curvature cases, for a Universe with a positive spatial curvature this regime is not its finite fate, and is ultimately followed by a recollapse of the Universe.

Unlike a recollapse, a transition from contraction to expansion (often called "bounce") being also possible, requires a specially imposed initial conditions [13]. These two characteristic features of a positive spatial curvature case - ultimate recollapse and existence of initial conditions, leading to a bounce - result in a complicated dynamics which in some situations may be chaotic.

It should be noted that the correct definition of chaos in General Relativity requires coordinate independent measures of chaos. For example, Lyapunov exponents do not satisfy this conditions because they can be set to zero by a simple coordinate transformations. One of possible invariants, the topological entropy measures how the number of periodic trajectories grows with respect to their complexity (see the definition in [4]). A non-zero topological entropy indicates the presence of chaos. Its calculation requires a detailed description of the set of periodical trajectories. A simpler possibility appears in transient chaotic system when there are at least two outcomes following a possible chaotic behavior. In this case we can plot basins of attraction in the initial condition space. A fractal nature of boundary between two basins indicates that dynamics is chaotic, while non-chaotic dynamics leads to smooth, regular boundaries (see [14] for a detailed description of this method). 


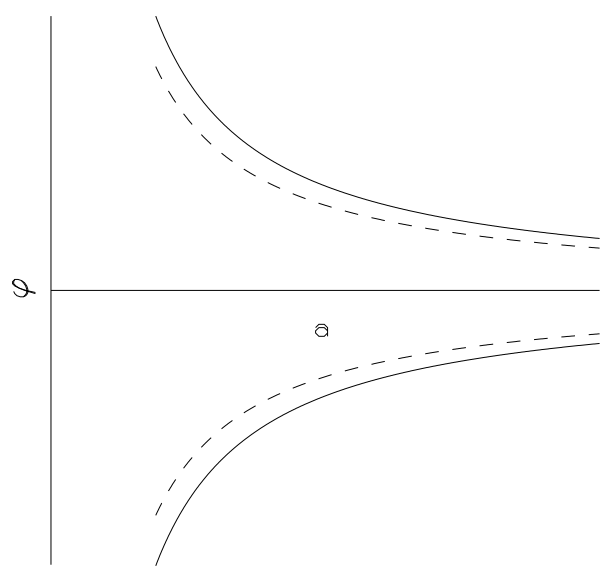

Figure 1. The boundary (5) (solid curves) for the potential $V=m^{2} \varphi^{2} / 2$. Points of maximal expansion are located between two dashed curves while bounces are possible in two narrow zones between solid and dashed curves.

\section{The nature of chaotic regime}

A very important property of the constraint equation (3) is that $\dot{a}^{2}$ and $\dot{\varphi}^{2}$ enter in the left-hand side with opposite signs. Rewriting (3) in the form

$$
-\frac{3 m_{P}^{2}}{8 \pi} \frac{\dot{a}^{2}}{a^{2}}+\frac{\dot{\varphi}^{2}}{2}=\frac{3 m_{P}^{2}}{8 \pi} \frac{1}{a^{2}}-V(\varphi)
$$

it is easy to see that

- There are no forbidden regions in the configuration space $(a, \varphi)$.

- The configuration space is divided into two regions - the region where the right-hand side of (4) is positive (and possible extrema of the scale factor are located), and the region where it is negative (where possible extrema of the scalar field are located).

The boundary between these two regions is the curve

$$
a^{2}=\frac{3}{8 \pi} \frac{m_{P}^{2}}{V(\varphi)}
$$

Zero-velocity points $(\dot{a}=\dot{\varphi}=0)$ of a trajectory, if they exist, should lie on this curve.

Consider points of maximal expansion and those of minimal contraction, i.e. the points, where $\dot{a}=0$ in more detail. They can exist only in the region where

$$
a^{2} \leq \frac{3}{8 \pi} \frac{m_{P}^{2}}{V(\varphi)}
$$

Using (1), we can show that possible points of maximal expansion $(\dot{a}=0, \ddot{a}<0)$ are localized inside the region

$$
a^{2} \leq \frac{1}{4 \pi} \frac{m_{P}^{2}}{V(\varphi)}
$$

while possible points of minimal contraction $(\dot{a}=0, \ddot{a}>0)$ lie outside this region (7) being at the same time inside the region (6) (see Fig. 1).

In [15] it was shown that the region of possible points of maximal expansion has quite a regular structure. In the part close to axis $a=0$ of this region there are points of maximal expansion, 
such that a trajectory, starting from them after some finite time reaches a point $a=0, \varphi=\infty$. In this point the equations of motion become singular and the trajectory can not be continued further in time. In General Relativity, this situation is called as a cosmological singularity. For bigger initial values of the initial scale factor one can see a narrow region where a trajectory experiences a bounce i.e. goes through the point of minimal contraction, after that the scale factor $a$ along the trajectory begins to grow. Increasing initial $a$ further, we obtain the region where a trajectory has a " $\varphi$-turn" i.e. has the extremum of the scalar field $\varphi$ and then falls into singularity. Then one has a region corresponding to trajectories having bounce after one oscillation in $\varphi$ and so on.

To avoid a misunderstanding, let us indicate once more what initial condition space we use. When we start from maximal expansion point, we fix one time derivative $(\dot{a}=0)$. So, to fix the initial condition completely, we need only to specify initial values of $a$ and $\varphi$. The initial $\dot{\varphi}$ is determined from the constraint equation (3), and our initial condition space becomes $(a, \varphi)$. We will study the structure of this space, investigating the location of points of maximal expansion, starting from which a trajectory has a bounce, but not the location of bounce itself. The $\varphi=0$ cross-section of regions, leading to bounce, are called as "bounce intervals".

Remembering that a trajectory describing an expanding universe must have a point of maximal expansion, we can apply this analysis further to bouncing trajectories after a bounce. Their second point of maximal expansion may lie either inside bounce regions, or between them. This fact generates a substructure of the region under consideration. The structure of subregions leading to two bounces repeats in general the structure of regions having at least one bounce, and so on and so forth. Continuing this process ad infinitum, we get the fractal zero-measure set of infinitely bouncing trajectories escaping the singularity.

If we distinguish two possible singular outcomes, $\varphi \rightarrow+\infty$ and $\varphi \rightarrow-\infty$, the method of basins boundaries can also be applied. Suppose that initial velocity in the point of maximal expansion is directed "up" (initial $\dot{\varphi}>0$ ). Then trajectories with even number of $\varphi$-turns will approach $\varphi \rightarrow+\infty$ singular point, while trajectories with odd number of $\varphi$-turns fall into $\varphi \rightarrow-\infty$ singularity. A boundary between these two basins is fractal, indicating the presence of chaos (see numerical examples in [4]).

In the first paper on chaos in FRW cosmology [3] D. Page have used another description. Instead of starting from a maximum expansion point, he began the analysis from zero-velocity point at the curve (5). Depending on the initial point, two different situations can be distinguished. Trajectories, going from this curve into the region (6) (the space between two solid hyperbolae in Fig. 1) have a point of maximal expansion soon after start, and then go towards a singularity. So, to prevent an almost immediate collapse, a trajectory must be directed into the region, where extrema of the scalar field are located (outside a solid hyperbola in Fig. 1). These two situations are separated by a particular trajectory, tangent to the curve (5). It means that this trajectory has

$$
\frac{\ddot{\varphi}}{\ddot{a}}=\frac{d \varphi}{d a}
$$

at the initial point. Here $\varphi(a)$ in the right-hand side is the equation of the curve (5). This point was first introduced by Page in [3] for massive scalar field potential $V(\phi)=m^{2} \phi^{2} / 2$. In this case

$$
\varphi_{\text {page }}=\sqrt{\frac{3}{4 \pi}} m_{P}, \quad a_{\text {page }}=1 / m,
$$

except for the trivial solution $\varphi=0, a=\infty$.

If at the initial point on the boundary (5)

$$
\frac{\ddot{\phi}}{\ddot{a}}<\frac{d \varphi}{d a}
$$




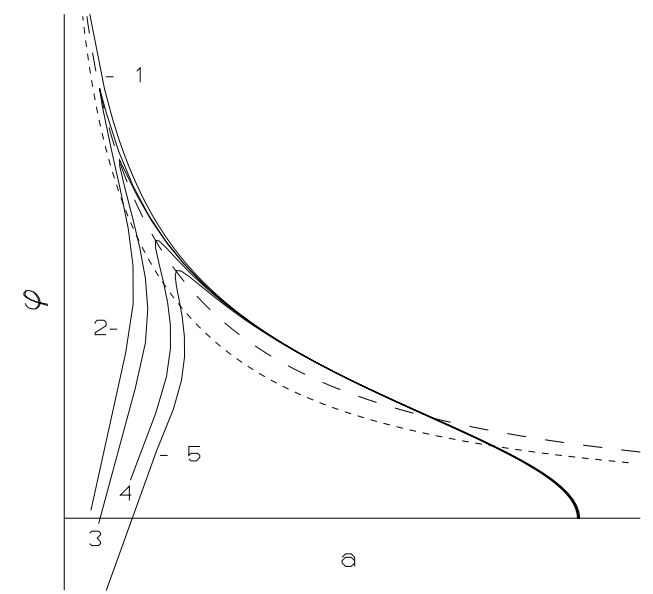

Figure 2. Example of trajectories with the initial conditions close to the boundary separating trajectories falling into $\varphi=+\infty$ (trajectory 1$)$ and $\varphi=-\infty$ (trajectories $2-5)$ singularities for the case $\varphi_{0}<\frac{\sqrt{3}}{4 \sqrt{\pi}} m_{P}$. This boundary is sharp, no fractal structure is present. Trajectories $2-5$ have a zigzag-like form, no periodical trajectories are present. The long-dashed line is the boundary (5), the short-dashed line separates zones of bounces from zones of maximal expansion points.

so that a trajectory is directed outside of the region (6), the Universe experiences a long enough expansion phase. For a massive scalar field (8) is satisfied if $\varphi>(3 / 4 \pi) m_{P}$. It should be noted, that a priori the significance of such trajectories for the chaotic structure is not evident, because we study now only those with a zero-velocity point. However, numerical data show that a large class of chaotic trajectories has zero-velocity points; in particular, all primary periodical orbits (i.e. having one bounce per period) contain such points (see examples in [4]).

With use of the equation of motion (1), (2) the criterion (8) gives for an arbitrary scalar field potential

$$
V(\varphi)>\sqrt{\frac{3 m_{P}^{2}}{16 \pi}} V^{\prime}(\varphi)
$$

The condition (9) may be treated as a restriction of local steepness of the function $V(\varphi)$. In can be easily seen that for power-law potentials there exists a value $\varphi_{\text {page }}$ such that for all $\varphi>\varphi_{\text {page }}$ the inequality (9) is satisfied. For steeper potentials the situation changes. For example, the potential $V(\varphi)=M_{0}^{4}\left(\cosh \left(\varphi / \varphi_{0}\right)-1\right)$ has a Page point only if $\varphi_{0}>\frac{\sqrt{3}}{4 \sqrt{\pi}} m_{P}$. In the opposite case the condition (9) is never satisfied and all trajectories starting from the curve (5) go into the region (6), experience the point of maximal expansion and fall into a singularity. Periodical trajectories with a zero-velocity point can not exist. It has been confirmed numerically that the chaos is absent in this case (periodical trajectories disappear, and the boundary between basins of attractions of $\varphi \rightarrow+\infty$ and $\varphi \rightarrow-\infty$ singularities becomes smooth) [7]. Although bounces with $\dot{a}=0, \ddot{a}>0$ are still possible, trajectories with bounces have a zigzag form, do not exit the region (6), and restore their direction to a singularity soon after the bounce (see Fig. 2).

For steeper potentials like

$$
V(\varphi)=M_{0}^{4}\left(\exp \left(\varphi^{2} / \varphi_{0}^{2}\right)+\exp \left(-\varphi^{2} / \varphi_{0}^{2}\right)-2\right)
$$

the condition (9) is definitely violated for large $\varphi$ but, depending on $\varphi_{0}$ it can be satisfied for intermediate $\varphi$. If so, zero-velocity points of periodical trajectories are located between two Page points. For the potential written above, Page points exist for $\varphi_{0}>0.905 m_{P}$. Our numerical 


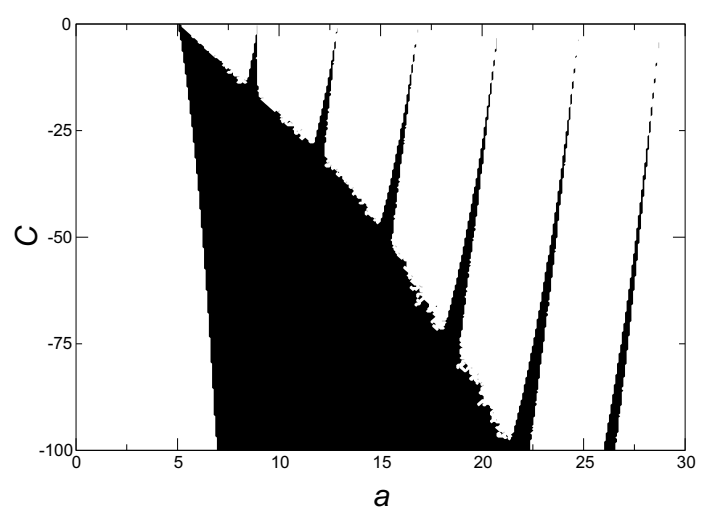

Figure 3. The $\varphi=0$ cross-section of the bounce intervals for the potential $V=m^{2} \varphi^{2} / 2$ and negative $C$. Consecutive merging of 5 first intervals can be seen in this range of $C$.

simulations show that the chaotic behavior exists if $\varphi_{0}>0.96 m_{P}$. So, we have a rather accurate and easily calculable condition for existence of the chaotic dynamics in the system (1), (2) (another example of a very steep potential see in [7]).

\section{Non-zero $C$}

Now we return to general properties of (1), (2) with an arbitrary value of the energy integral $C$ in (3). One particular case of this problem have already been investigated. Namely, it can be shown that our dynamical system with positive $C$ if formally equivalent to the system describing a scalar field in the presence of a pressure-less matter. A detailed study of this case for massive scalar field potential $V(\phi)=m^{2} \varphi^{2} / 2$ have been done in [6]. The structure of the periodical trajectories becomes more complicated in comparison with $C=0$ case. First of all, the number of bounce interval becomes finite and diminishes with increasing $C$. Besides, we have a nontrivial set of selection rules, which should be satisfied for any allowed sequence of intervals along an arbitrary trajectory (in general, the bigger is the ordinal number of the first interval, the smaller should be a maximum possible number of the second one). One particular example of such rules (simplified a little in comparison with those seen in computer simulations) was described in [6] with calculation of corresponding topological entropy. If $C$ increases, the topological entropy decreases (the width of initial space regions leading to bounce decreases also), and for $C m>0.023 m_{P}^{2}$ the chaos disappears.

For positive $C$ this situation is general - chaos disappears for energy integral exceeding some critical value which depends on particular form of the potential. A negative $C$ alter the nature of chaos in a different way. Though there are no direct physical applications of a system (1), (2) with a negative $C$, mathematical properties of this case are very interesting. Besides, there are dynamical systems of the form similar to (1), (2) with physically admissible negative $C$ - they appear in brane cosmology [16].

When $C$ goes to the range of negative values, the width of the bounce regions steadily increases. As an example, we continue to investigate the massive scalar field case. One of our numerical plots is shown in Fig. 3. We plotted the $\varphi=0$ cross-section of bounce regions depending on $C$. This plot represents a situation, qualitatively different from studied previously for positive or zero $C$. Namely, the bounce intervals can merge.

Let us see more precisely what does it mean. For $C>-20$ the picture is qualitatively the same as for $C=0$ - trajectories from 1-st interval have a bounce with no $\varphi$-turns before it, trajectories which have initial point of maximal expansion between 1-st and 2-nd intervals fall into a singularity after one $\varphi$-turn, those from 2 -nd interval have a bounce after $1 \varphi$-turn and 
so on. For $|C|$ a bit larger than the first merging value, the 2-nd interval contains trajectories with $2 \varphi$-turns before bounce, the space between 1-st interval (this interval is now a product of two merged intervals) and the 2-nd interval contains trajectories falling into a singularity after two $\varphi$-turns. There are no trajectories falling into a singularity with exactly one $\varphi$-turn. Trajectories from the 1-st interval can experience now a complicated chaotic behavior, which can not be described in a way, similar to the $C=0$ case.

With $C$ decreasing further, the process of interval merging continues leading to a growing chaotization of trajectories. When $n$ intervals are merged together, only trajectories with at least $n$ oscillations of the scalar field before falling into a singularity are possible. Those having exactly $n \varphi$-turns have their initial point of maximal expansion between the 1-st interval and the 2-nd one (the second interval now contains trajectories having a bounce after $n \varphi$-turns). For initial values of the scale factor larger then those from the 2-nd interval, the regular quasiperiodic structure described above is restored. On the other hand, initial values from the 1-st interval lead to a very complicated structure of chaotic trajectories. Significant part of them do not fall into a singularity for an arbitrary long time of computer simulations. This fact indicates the presence of a strong chaotic regime, which is qualitatively more complex than the regime corresponding to a rather regular fractal structure described in the previous section. The structure of strong chaos requires further investigations.

\section{$5 \quad$ Less steep potentials}

So far we have found that a positive $C$ as well as steep potentials are less favorable for chaos. To prove these dependencies further we consider potentials, less steep than the quadratic one. We will investigate a common family of potentials having power-low asymptotic - DamourMukhanov potentials [17]. They were originally introduced to show a possibility for the Universe to have an inflationary expansion without the slow-roll regime. The explicit form of DamourMukhanov potential is

$$
V(\varphi)=\frac{M_{0}^{4}}{q}\left[\left(1+\frac{\varphi^{2}}{\varphi_{0}^{2}}\right)^{q / 2}-1\right] .
$$

with three parameters $M_{0}, q$ and $\varphi_{0}$.

For $\varphi \ll \varphi_{0}$ the potential looks like the massive one with the effective mass $m_{\text {eff }}=M_{0}^{2} / \varphi_{0}$. In the opposite case of large $\varphi$ it grows like $\varphi^{q}$.

Changing $M_{0}$ leads only to rescaling $a$ and does not alter the type of chaos. So, we have a two-parameter $\left(q\right.$ and $\varphi_{0}$ ) family of potentials with different chaotic properties. The main result of our numerical studies is that the event, described in the previous section - existence of a strong chaos regime - takes place also for this family of gently sloping potentials even with $C=0$. Numerical studies show the following picture (see Fig. 4): for a small enough $q$ there exists a corresponding critical value of $\varphi_{0}$ such that for $\varphi_{0}$ less than the critical one, the strong chaotic regime exists. Increasing $q$ corresponds to decreasing the critical $\varphi_{0}$.

Since this regime is absent for quadratic and steeper potentials, $q$ must at least be less than 2 . We can see clearly the strong chaotic regime for $q<1.24$. Near this value the critical $\varphi_{0}$ decreases very sharply.

To study further the existence of the strong chaotic regime in gently sloping potentials we consider another family of potentials with the same asymptotic for large $\varphi$ and another behavior for small $\varphi$ :

$$
V(\varphi)=\frac{M_{0}^{4}}{q}\left[\left(1+\frac{\varphi^{4}}{\varphi_{0}^{4}}\right)^{q / 4}-1\right] .
$$




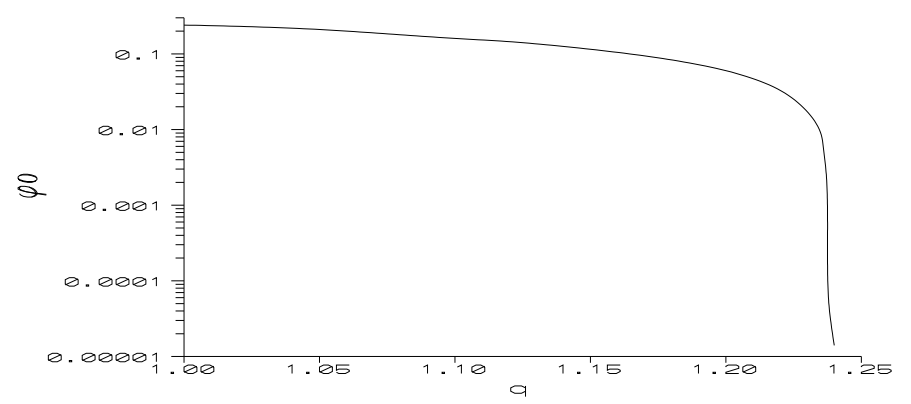

Figure 4. The value $\varphi_{0}$ of the potential (10) corresponding to the first merging of the bounce intervals depending on power index $q$.

For small $\varphi$ the potential $V(\varphi) \sim \lambda \varphi^{4}$ with $\lambda=M_{0}^{4} /\left(4 \varphi_{0}^{4}\right)$, though for large $\varphi$ we still have $V(\varphi) \sim \varphi^{q}$. Our numerical studies have shown that the critical $\varphi_{0}$ in this case is close to the number obtained for Damour-Mukhanov potentials and has the same asymptotic value $\sim 1.24$. We conclude that existence of the strong chaos depends mainly on the parameter $q$.

So far we found that decreasing steepness of the potential acts as decreasing $C$. The opposite is also true - if we start from a strong chaos for some suitable gently sloping potential with $C=0$, and then allow $C$ to increase, the bounce intervals consecutively separate, strong chaos disappears and with further increasing $C$ the chaotic regime disappears completely [18].

\section{Potentials, steeper than quadratic one with negative $C$}

In the area of potentials steeper than the quadratic one and negative $C$ we can see two tendencies acting in opposite directions. We begin with presentation of our numerical results on the strong chaotic regime. We already know that the strong chaos exists for $V=m^{2} \varphi^{2} / 2$ with sufficiently large negative $C$. On the other hand, our numerical analysis have shown that for the potential $V(\varphi) \sim \varphi^{4}$ there is no strong chaos for any $C$. In order to find the biggest power index allowing the strong chaos regime, we use the potential family (11). Our numerical data lead to conclusion that large enough negative $C$ could produce the strong chaos for $q<2.15$.

As for possibility for chaotic regime itself, the value of $C$ alters the critical $\varphi_{0}$ in the exponentially steep potentials $V \sim\left(\cosh \left(\varphi / \varphi_{0}\right)-1\right)$, studied in the Section 3. Negative $C$ makes the chaos possible for a wider range of $\varphi_{0}$. In the limit $C \rightarrow-\infty$ the analog of Page point equation does not depend on $C$ :

$$
\sqrt{3} V(\varphi)>\sqrt{\frac{m_{P}^{2}}{4 \pi}} V^{\prime}(\varphi)
$$

and, so, the chaos is absent if $\varphi_{0}$ is less than $m_{P} / \sqrt{12 \pi}$ for any value of $C$.

\section{Conclusions}

We have investigated possible regimes for the dynamical system describing a FRW cosmological model with a scalar field, and the generalization of this system to a non-zero "energy parameter" $C$. The qualitative features of this dynamics depend on the steepness of the scalar field potential $V(\phi)$ and the value of the "energy parameter". The general property is that the chaotic behavior is more significant for less steep potentials and lower values of $C$. The known description of chaos in FRW cosmology with a massive scalar field $[4,15]$ appears to be typical for power-law potentials. In this case only trajectories starting from narrow quasi-parallel zones in 
the initial condition space $(a, \varphi)$ from the point of maximal expansion do not fall into a singularity at the first contraction stage. The full description of possible periodic trajectories can be done easily using a sufficiently simple coding [4].

For less steep potential, however, these zones can intersect and merge, and the behavior of trajectories becomes more complex. In FRW cosmology $(C=0)$ this strong chaotic regime can appear if asymptotically $V(\phi) \sim \phi^{p}$ with $p<1.24$, in the general case corresponding power index can reach 2.15 if $C$ is negative and large.

For potentials steeper than power-law the chaotic regime can disappear. We have studied exponentially steep potential in the form $V(\phi)=M_{0}^{4}\left(\cosh \left(\phi / \phi_{0}\right)-1\right)$. The condition for chaos is $\phi_{0}>4 m_{P} / \sqrt{3}$ for $C=0$. Negative $C$ makes this critical value of $\phi_{0}$ lower. For $C \rightarrow-\infty$ the critical $\phi_{0}$ tends to $m_{P} / 2 \sqrt{3 \pi}$, being 1.5 times less then for the $C=0$ case.

A sufficiently large positive $C$ kills chaos for an arbitrary potential $V(\phi)$.

\section{A Appendix}

In this appendix we briefly describe several dynamical systems which are generalization of (1), (2) in different cosmological scenarios. In all examples we consider only $C=0$ case.

\section{A.1 Cosmological constant}

If we ad a constant term $L$ (often written in the form $L=\left(m_{P}^{2} / 8 \pi\right) \Lambda$, where $\Lambda$ is the cosmological constant) to a potential $V(\varphi)$, the cosmological dynamics changes significantly. A new late-time attractor (called the DeSitter regime) with $H \rightarrow \sqrt{\Lambda / 3}$ (and, correspondingly, $a \sim \exp (H t)$ ) appears. Trajectories, reaching this attractor have no maximal expansion point. Large enough $\Lambda$ leads to a situation when all bouncing trajectories falls into the DeSitter regime, and chaotic behavior disappears [5]. For a massive scalar field this happens if $\Lambda$ is larger than $\sim 0.3 m^{2}$. It should be noticed, that the Page points for the potential $V(\varphi)=L+m^{2} \varphi^{2} / 2$ disappear for $\Lambda=0.75 m^{2}$ [7], so the Page points criterion does not work well in this case.

\section{A.2 Complex scalar field}

For description of a scalar field with non-zero charge a formalism of complex scalar field is used. The most natural representation of the complex scalar field has the form

$$
\phi=x \exp (i \theta)
$$

where $x$ is the absolute value of the field while $\theta$ is its phase. This phase is a cyclical variable corresponding to the conserved quantity - a classical charge $Q \equiv a^{3} x^{2} \dot{\theta}$. The equations of motion are [19]

$$
\frac{m_{P}^{2}}{16 \pi}\left(\ddot{a}+\frac{\dot{a}^{2}}{2 a}+\frac{1}{2 a}\right)+\frac{a \dot{x}^{2}}{8}-\frac{a V(x)}{4}+\frac{Q^{2}}{4 a^{5} x^{2}}=0
$$

and

$$
\ddot{x}+\frac{3 \dot{x} \dot{a}}{a}+V^{\prime}(x)-\frac{2 Q^{2}}{a^{6} x^{3}}=0
$$

with the first integral

$$
-\frac{3}{8 \pi} m_{P}^{2}\left(\dot{a}^{2}+1\right)+\frac{a^{2}}{2}\left(\dot{\varphi}^{2}+2 V(\varphi)\right)+\frac{Q^{2}}{2 a^{4} x^{2}}=0 .
$$

The effect of terms containing the charge $Q$ is similar to the influence of positive $C$ chaotic behavior disappears for a sufficiently large $|Q|$. For $V=m^{2} \varphi^{2} / 2$ it happens if $|Q| m^{2}>$ $0.056 m_{P}^{2}[6]$ 


\section{A.3 Brane Universe}

The equation of motion for a Randall-Sundrum brane Universe [20] in high-energy regime has the form [21]

$$
\begin{aligned}
& \frac{\dot{a}^{2}}{a^{2}}+\frac{1}{a^{2}}=\frac{\kappa^{4}}{36}\left(\frac{1}{2} \dot{\varphi}^{2}+V\right)^{2}+\frac{C}{a^{4}}, \\
& \frac{\ddot{a}}{a}+\frac{2 \dot{a}^{2}}{a^{2}}+\frac{2}{a^{2}}=\frac{\kappa^{4}}{48}\left(4 V^{2}-\dot{\varphi}^{4}\right)+\frac{C}{a^{4}}, \\
& \ddot{\varphi}=-3 \frac{\dot{a}}{a} \dot{\varphi}-V^{\prime}(\varphi),
\end{aligned}
$$

where $\kappa^{2}=8 \pi / M_{(5)}^{3}, M_{(5)}$ is a fundamental 5 -dimensional Planck mass, $C$ is an integration constant.

In the case of $C=0$ the condition (8) is

$$
\frac{\kappa^{4}}{36} V(\varphi)^{3}>\left(\frac{d V(\varphi)}{d \varphi}\right)^{2}
$$

It is easy to see that in this case the Page points exist for exponential and even steeper than exponential potentials. They disappear only for potentials in the form of infinite potential wall. The critical case now is the potential

$$
V(\varphi)=\frac{A}{\left(\varphi-\varphi_{0}\right)^{2}}
$$

and chaos disappears for $A<9 M_{(5)}^{6} /\left(4 \pi^{2}\right)[22]$.

\section{A.4 Anisotropic Universe}

The next possibility to generalize equations (1), (2), apart from modifying a scalar field or effective theory of gravity (as have been done in the brane worlds scenario) is to consider a broader than FRW class of metrics. If we lift the assumption of spatial isotropy, the evolution of the Universe will be described by three different scale factors $a, b$ and $c$. The Einstein equations for a closed Universe with a massive scalar field take the form

$$
\begin{aligned}
& \frac{(\dot{a} b c)^{\cdot}}{a b c}+\frac{1}{2 a^{2} b^{2} c^{2}}\left[a^{4}-\left(b^{2}-c^{2}\right)^{2}\right]=\frac{m^{2}}{4} \varphi^{2}, \\
& \frac{(a \dot{b} c)^{\circ}}{a b c}+\frac{1}{2 a^{2} b^{2} c^{2}}\left[b^{4}-\left(a^{2}-c^{2}\right)^{2}\right]=\frac{m^{2}}{4} \varphi^{2}, \\
& \frac{(a b \dot{c})^{\circ}}{a b c}+\frac{1}{2 a^{2} b^{2} c^{2}}\left[c^{4}-\left(a^{2}-b^{2}\right)^{2}\right]=\frac{m^{2}}{4} \varphi^{2}, \\
& \ddot{\varphi}+\left(\frac{\dot{a}}{a}+\frac{\dot{b}}{b}+\frac{\dot{c}}{c}\right) \dot{\varphi}+m^{2} \varphi=0
\end{aligned}
$$

with the first integral

$$
\frac{1}{2} \dot{\varphi}^{2}+\frac{m^{2}}{2} \varphi^{2}=2\left(\frac{\dot{a}}{a} \frac{\dot{b}}{b}+\frac{\dot{a}}{a} \frac{\dot{b}}{b}+\frac{\dot{b}}{b} \frac{\dot{c}}{c}\right)+\frac{1}{a^{2}}+\frac{1}{b^{2}}+\frac{1}{c^{2}}-\frac{a^{4}+b^{4}+c^{4}}{2 a^{2} b^{2} c^{2}} .
$$

This system (in the case of empty Universe, where the scalar field is absent) is well known due to chaotic oscillations of scale factors at the contraction stage, when the overall volume $v=a b c$ decreases monotonically - so called Belinsky-Lifshits-Khalatnikov chaos [23]. On the other 
hand, chaotic oscillations of the volume itself due to presence of a scalar field have attracted less attention. It is known that in general a spatial anisotropy destroys bouncing behavior, and bounce can survive only for small initial deviation from isotropy. No anisotropic periodical trajectories have been found yet [24].

As a final remark, it should be noticed that modern modifications of General Relativity, elaborated for quantization of gravity can modify cosmological dynamics significantly in their corresponding semi-classical regimes. For two interesting examples we note a new non-singular cosmological attractor in String Gravity [25, 26, 27] and an ultimate bounce independent of initial conditions and a scalar field potential in Loop Quantum Cosmology [28] (for general descriptions of these theories, see, for example, [29, 30, 31, 32]).

\section{Acknowledgments}

Author is grateful to A.Yu. Kamenshchik, I.M. Khalatnikov, S.V. Savchenko, S.A. Pavluchenko, S.O. Alexeyev, V.O. Ustiansky, P.V. Tretyakov and Parampreet Singh for discussions and collaboration in studies of chaos in a scalar field cosmology.

[1] Kantz H., Grassberger P., Repellers, semiattractors and long lived chaotic transients, Phys. D, 1985, V.17, 75-93.

[2] Gaspard P., Rice S.A., Scattering from a classically chaotic repellor, J. Chem. Phys., 1989, V.90, $2225-2241$.

[3] Page D.N., A fractal set of perpetually bouncing Universes?, Classical Quantum Gravity, 1984, V.1, $417-441$.

[4] Cornish N.J., Shellard E.P.S., Chaos in quantum cosmology, Phys. Rev. Lett., 1998, V.81, 3571-3574.

[5] Kamenshchik A.Yu., Khalatnikov I.M., Toporensky A.V., Simplest cosmological model with the scalar field. 2. Influence of cosmological constant, Internat. J. Modern Phys. D, 1998, V.7, 129-138, gr-qc/9801082.

[6] Kamenshchik A.Yu., Khalatnikov I.M., Savchenko S.V., Toporensky A.V., Topological entropy for some isotropic cosmological models, Phys. Rev. D, 1999, V.59, 123516, 28 pages, gr-qc/9809048.

[7] Toporensky A.V., Chaos in closed isotropic cosmological models with steep scalar field potentials, Internat. J. Modern Phys. D, 1999, V.8, 739-750, gr-qc/9812005.

[8] Linde A.D., Particle physics and inflationary cosmology, Harwood Academic, 1990.

[9] Belinsky V.A., Grishchuk L.P., Zeldovich Ya.B., Khalatnikov I.M., Inflationary stages in cosmological models with scalar fields, JETP, 1985, V.89, 346-360 (in Russian).

[10] Belinsky V.A., Khalatnikov I.M., On the degree of generality of inflationary solutions in cosmological models with a scalar field, Sov. Phys. JETP, 1987, V.93, 441-472.

[11] Belinsky V.A., Ishihara H., Khalatnikov I.M., Sato H., On the degree of generality of inflation in Friedman cosmological models with a massive scalar field, Progr. Theoret. Phys., 1988, V.79, 676-684.

[12] Turner M., Coherent scalar field oscillations in an expanding Universe, Phys. Rev. D, 1983, V.28, $1243-1256$.

[13] Starobinsky A.A., On a nonsingular isotropic cosmological model, Sov. Astron. Lett., 1978, V.4, 82-84.

[14] Cornish N., Levin J., Chaos, fractals and inflation, Phys. Rev. D, 1996, V.53, 3022-3032, astro-ph/9510010.

[15] Kamenshchik A.Yu., Khalatnikov I.M., Toporensky A.V., Simplest cosmological model with the scalar field, Internat. J. Modern Phys. D, 1997, V.6, 673-692, gr-qc/9891064.

[16] Maartens R., Cosmological dynamics on the brane, Phys. Rev. D, 2000, V.62, 084023, 24 pages, hepth/0004166.

[17] Damour T., Mukhanov V.F., Inflation without slow roll, Phys. Rev. Lett., 1998, V.80, 3440-3443, grqc/9712061.

[18] Pavluchenko S.A., Toporensky A.V., Chaos in FRW cosmology with gently sloping scalar field potential, Gravitation and Cosmology, 2000, V.6, 241-245, gr-qc/9911039.

[19] Kamenshchik A.Yu., Khalatnikov I.M., Toporensky A.V., Complex inflaton field in quantum cosmology, Internat. J. Modern Phys. D, 1997, V.6, 649-672, gr-qc/9801039.

[20] Randall L., Sundrum R., An alternative to compactification, Phys. Rev. Lett., 1999, V.83, 4690-4693, hep-th/9906064.

[21] Binetruy P., Deffayet C., Ellwanger U., Langlois D., Brane cosmological evolution in a bulk with cosmological constant, Phys. Lett. B, 2000, V.477, 285-291, hep-th/9910219. 
[22] Toporensky A.V., Tretyakov P.V., Ustiansky V.O., New properties of scalar field dynamics in brane isotropic cosmological models, Astron. Lett., 2003, V.29, 1-5, gr-qc/0207091.

[23] Belinsky V.A., Khalatnikov I.M., Lifschitz E.M., Oscillators approach to a singular point in the relativistic cosmology, Adv. Phys., 1970, V.19, 525-573.

[24] Toporensky A.V., Ustiansky V.O., Dynamics of Bianchi IX universe with massive scalar field, gr-qc/9907047.

[25] Antoniadis I., Rizos J., Tamvakis K., Singularity-free cosmological solutions of the superstring effective action, Nucl. Phys. B, 1994, V.415, 497-514, hep-th/9305025.

[26] Rizos J., Tamvakis K., On the existence of singularity-free solutions in quartic gravity, Phys. Lett. B, 1994, V.326, 57-61, gr-qc/9401023.

[27] Alexeyev S.O., Toporensky A.V., Ustiansky V.O., Non-singular cosmological models in string gravity with constant dilaton and second order curvature corrections, Classical Quantum Gravity, 2000, V.17, 2243-2254, gr-qc/9912071.

[28] Singh P., Toporensky A.V., Big crunch avoidance in $k=1$ semiclassical loop quantum cosmology, Phys. Rev. D, 2004, V.69, 104008, 5 pages, gr-qc/0312110.

[29] Damour T., Polyakov A., String theory and gravity, Gen. Relativity Gravitation, 1994, V.26, 1171-1176, gr-qc/9411069.

[30] Bento M., Bertolami O., Cosmological solutions of higher-curvature string effective theories with dilaton, Phys. Lett. B, 1996, V.368, 198-201, gr-qc/9503057.

[31] Bojowald M., The semiclassical limit of loop quantum cosmology, Classical Quantum Gravity, 2001, V.18, L109-L116, gr-qc/0105113.

[32] Bojowald M., Isotropic loop quantum cosmology, Classical Quantum Gravity, 2002, V.19, 2717-2742, grqc/0202077. 